\title{
AUTOMATIC AND REAL-TIME LOCOMOTION MODE RECOGNITION OF A HUMANOID ROBOT*
}

\author{
JOANA FIGUEIREDO $^{a}$, DIOGO GONÇALVES $^{a}$ \\ ${ }^{a}$ Center for MicroElectroMechanical Systems, University of Minho \\ 4800-058 Guimarães, Portugal
}

JUAN C. MORENO ${ }^{b \dagger}$

${ }^{b}$ Neural Rehabilitation Group, Cajal Institute, Spanish National Research Council 28002 Madrid, Spain

\section{CRISTINA P. SANTOS ${ }^{a}$}

\begin{abstract}
Real-time locomotion mode recognition can potentially be applied in the gait analysis as a diagnostic tool or a strategy to control the robotic motion. This research aimed the development of an automatic, accurate and time-effective tool to recognize, in real-time, the locomotion mode that is being performed by a humanoid robot. The proposed strategy should also be general to different walkers and walking conditions. For these purposes, we designed a strategy to identify, in an offline phase, the suitable features and classification models for the real-time recognition. We explored several classification models based on two machine learning approaches using the features previously selected by principal component analysis and genetic algorithm (GA). The validation was carried out for distinct walking directions and speeds of DARwIn-OP. The offline analysis suggests that the most skilled models are the ones created by weighted k-nearest neighbors (KNN), fine KNN and cubic support vector machine using 2 features selected by GA. Results from the realtime implementation highlight that weighted $\mathrm{KNN}$ exhibits a higher recognition performance (accuracy $>99.15 \%$ ) and a lower elapsed time in the recognition process $(89$ $\mathrm{ms}$ ) comparatively to the state-of-the-art. The proposed recognition tool showed to be costeffective, and highly accurate for the real-time gait analysis at different walking conditions.
\end{abstract}

\section{Introduction}

Recent studies have argued that the automatic recognition of locomotion mode has potential to be applied as an assessment tool of locomotion performance, as

\footnotetext{
* This work is supported by the FCT - Fundação para a Ciência e Tecnologia - with the scholarship reference SFRH/BD/108309/2015, the reference project UID/EEA/04436/2013, by FEDER funds through the COMPETE 2020 - Programa Operacional Competitividade e Internacionalização (POCI) - with the reference project POCI-01-0145-FEDER-006941.

$\dagger$ Work partially supported by grant RYC-2014-16613 by Spanish Ministry of Economy and Competitiveness.
} 
well as a strategy to contribute to real-time information for the guided-motion control demanded on smart robotic systems [1].

Few studies have developed tools to recognize, in real-time, the locomotion mode. Pattern-recognition techniques based on the electromyography (EMG) signal have been implemented [2], [3]. However, EMG has some drawbacks comparatively to other biomechanical sensors, such as the user's fatigue, sweating, electrodes shift that can degrade the classification performance over time [2], [4]. To avoid these limitations, other studies [4], [5] considered that gait dynamics from inertial sensors or the association of diverse data [6] would conduct a more comprehensive and robust automatic recognition scheme.

The strategies proposed for the locomotion mode recognition are essentially based on optimal thresholds implemented through finite state machines (FSMs) [1], [4], [7], or machine learning approaches, such as support vector machines (SVMs) [3], [8], [9], dynamic Bayesian network [2], linear discriminant analysis [2], [9], and artificial neuronal network (ANN) [8]. The major advantage of FSMs comparatively to the machine learning approaches is their simplicity [1]. Machine learning approaches for the human movement recognition, namely SVMs, stand out by their generalization ability even using a limited training data set [10], [11], and the advantage of easily incorporating newly available data [8]. Nevertheless, the works proposed in the literature present high timing delays (ranging from 300 to $650 \mathrm{~ms}$ [3], [5]) for the recognition of a new locomotion mode, compromising the application of the proposed tools in the real-time scenario.

This study aims to develop an intelligent and a time-effective strategy to recognize, in real-time, the locomotion mode that is being performed by a humanoid robot. We also consider that the proposed strategy should be general to different users and walking conditions. For this purpose, we applied two machine learning approaches (SVM and k-nearest neighbors (KNN)) due to their generalization ability and robustness in the recognition of newly data. In fact, few studies in the literature focused on this last issue [11]. The SVM and KNN were explored regarding the complexity to make a decision in order to investigate the trade-off between the lower complexity of KNN and the high efficiency of SVM. Based on this trade-off, we disclosed which classifier is more skilled to evaluate the gait function and to support the control of robotic devices. 


\section{Methods}

\subsection{Proposed Strategy and Implementation}

The strategy designed and implemented to recognize the actual locomotion modes is depicted in Figure 1.a). This strategy is structured into two phases, which are processed first offline and then in real-time. The offline phase aims to select the most significant features that characterize each locomotion mode and to create the classification model for the recognition process. On the other hand, the real-time phase focuses on the real-time locomotion mode recognition using the classification model created in the offline phase and the gait data recorded in realtime. The proposed strategy was implemented during the walking of the DARwInOP humanoid robot.

As pointed out in Figure 1.a), the first stage involves the sensing of gait dynamics, in particular, joint angles and the ground reaction forces from the inertial and force sensors embedded on DARwIn-OP, respectively. Using the data acquired, we determined 6 temporal parameters (stride duration, step duration, strides per minute, cadence, stance duration, and swing duration) and 15 kinematic parameters (range of motion (ROM) and angle at the heel strike and toe-off events for the hip, knee, metatarsal and ankle joints, and thigh) per observation. These features constitute the biomechanical parameters that characterize each locomotion mode. Posteriorly, we normalized the 21 features through the $z$-score method to make the classification model more accurate due to the usage of standardized features [8]. In the real-time phase, the features were computed after the DARwIn-OP had walked one stride since part of the interval features can only be determined when at least one stride is performed.

We designed that the fourth stage of the offline phase aims the selection of the most significant features by applying linear principal component analysis (PCA) and genetic algorithm (GA) through Matlab®. The input parameters of GA were specified based on a trade-off between the performance and the computational cost of this stage. These parameters were defined as: population size (100 individuals); maximal number of iterations to perform (100); and, the number of hold individuals among interactions (2). Additionally, we defined that the fitness function of GA aimed the minimization of classification error exhibited by the classification models (SVM or KNN), which was iteratively being created with the features previously selected by the GA. To assess the generation ability of each classifier to classify new instances, we performed a 10-folds CV method that is commonly applied in the literature [12]. 
The machine learning approaches used in this work were also implemented by means of Matlab®. For the SVM, we implemented 6 different kernels, which led to the following 6 classification models: Coarse Gaussian SVM; Fine Gaussian SVM; Medium Gaussian SVM; Quadratic SVM; Cubic SVM; and, Linear SVM. In this work, we extended the SVM for multiclass classification by means "one-against-one" (OAO) since the "one-against-all" approach has shown to be more complex in making a decision and present an exhaustive training process. Regarding the KNN, we also implemented 6 different classification models (Coarse KNN, Medium KNN, Weighted KNN, Fine KNN, Cosine KNN, Cubic KNN) by specifying different distance metrics (Euclidean, Cosine, and Minkowski) and number of neighbors (10 neighbors in 5 models and 1 neighbor for Fine KNN). Overall, we implemented 12 classification models per each feature selection method, and per each recognition process.

To facilitate the use of the proposed classification models, we also developed in Matlab® a user interface, which is illustrated in Figure 1.b). For this purpose, we established a robust real-time internet communication (TCP communication) between the recognition tools implemented in Matlab® and the walker studied in Webots $^{\mathrm{TM}}$. The user interface also indicates the locomotion mode that is being performed by DARwIn-OP, the elapsed time in the recognition, as well as the performance of the selected model.

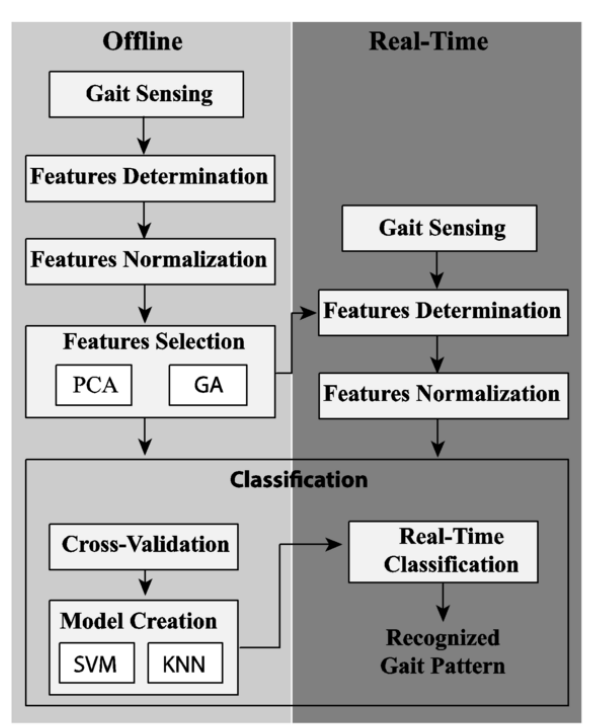

a)

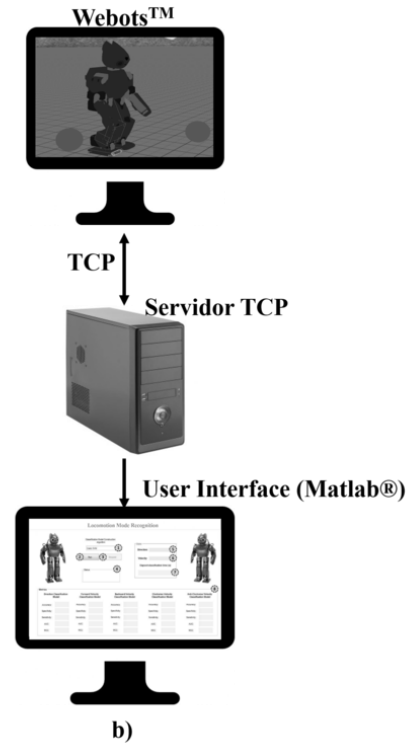

b)

Figure 1. a) Proposed strategy for locomotion mode recognition. b) TCP communication between DARwIn-OP in the simulated environment (Webots ${ }^{\mathrm{TM}}$ ) and the user interface (Matlab®). 


\subsection{Validation Conditions}

The proposed strategy was validated in real-time during the level-ground walking of DARwIn-OP in a simulated environment (Webots ${ }^{\mathrm{TM}}$ showed in Figure 1.b). The gait patterns of DARwIn-OP were perturbed with noise to create a walking environment that more closely resembles the one observed in the real-world.

The performance of the implemented strategy was investigated in four distinct walking directions: forward walking; backward walking; turning left; and, turning right. For each walking direction, we also recognized three different walking speeds of the DARwIn-OP: low speed $(<0.6 \mathrm{~m} / \mathrm{s})$; moderate speed (from 0.6 to $0.8 \mathrm{~m} / \mathrm{s})$; and, high speed $(>0.8 \mathrm{~m} / \mathrm{s})$. For the offline phase, we conducted 3 trials for each locomotion mode.

\section{Results and Discussion}

\subsection{Results of Offline Phase}

From the gait sensing stage performed in offline phase, we acquired 1020 observations for the different walking directions and 135, 142, 333, and 411 observations when the DARwIn-OP walked at different speeds on forward, backward, turning right, and turning left conditions, respectively. We included different sizes of observations among the different locomotion modes to investigate the performance of the implemented classification models during the recognition of the unbalanced gait data.

Overall, we developed 5 different real-time recognition tools for the locomotion mode of DARwIn-OP; 1 to recognize the walking direction (4 walking directions that correspond to 4 classes), and the other 4 to recognize the walking speed ( 3 walking speeds that correspond to 3 classes) for each walking direction previously detected. Thus, 5 types of multiclass classifiers (each one created from 2 features selection methods combined with 12 classification models) were investigated in real-time, and previously created in offline.

To investigate the performance of each recognition tool, we performed an objective analysis according to five metrics: accuracy, sensitivity, specificity, area under the curve (AUC), and Matthews correlation coefficient (MCC). Note that we introduced MCC due to its ability to deal with unbalanced observations, such the ones used in this work. For space constraints, we just disclose in Table 1 the performance achieved in the recognition of walking direction when the best feature selection method was applied. Analyzing Table 1, we can indicate that all recognition tools implemented are highly accurate (accuracy $>93.1 \%$ ), specific (specificity $>98.2 \%$ ) and robust. In fact, the best recognition tool developed in 
this work showed a higher performance than the other similar tools proposed in the literature [8], [9], [13]. Moreover, the high values of MCC (>97\%) suggest that the machine learning methods proposed are robust in recognition even when nonlinear, multidimensional and unbalanced data are used.

Table 1. Performance obtained in offline recognition of walking direction.

\begin{tabular}{|c|c|c|c|c|c|c|c|}
\hline \multirow{2}{*}{$\begin{array}{l}\text { Machine } \\
\text { learning }\end{array}$} & \multicolumn{2}{|c|}{ Features selection } & \multirow{2}{*}{$\begin{array}{c}\text { Accuracy } \\
(\%)\end{array}$} & \multirow{2}{*}{$\begin{array}{c}\text { Specificity } \\
(\%)\end{array}$} & \multirow{2}{*}{$\begin{array}{c}\text { Sensibility } \\
(\%)\end{array}$} & \multirow{2}{*}{$\begin{array}{c}\mathbf{A U C} \\
(\%)\end{array}$} & \multirow{2}{*}{$\begin{array}{c}\mathrm{MCC} \\
(\%)\end{array}$} \\
\hline & $\#$ & Method & & & & & \\
\hline $\begin{array}{l}\text { Cosine } \\
\text { KNN }\end{array}$ & 3 & GA & 98.75 & 98.71 & 98.61 & 98.98 & 97.94 \\
\hline Cubic KNN & 2 & GA & 98.04 & 99.43 & 98.692 & 98.91 & 97.02 \\
\hline $\begin{array}{l}\text { Weighted } \\
\text { KNN }\end{array}$ & 2 & GA & 99.87 & 99.92 & 99.31 & 99.15 & 99.22 \\
\hline $\begin{array}{l}\text { Coarse } \\
\text { KNN }\end{array}$ & 2 & GA & 93.12 & 98.01 & 95.23 & 98.24 & 90.69 \\
\hline $\begin{array}{l}\text { Medium } \\
\text { KNN }\end{array}$ & 2 & GA & 98.17 & 99.46 & 98.77 & 98.92 & 97.20 \\
\hline Fine KNN & 2 & GA & 99.06 & 99.42 & 98.92 & 98.95 & 98.93 \\
\hline Linear SVM & 21 & No & 99.06 & 99.18 & 98.92 & 98.98 & 98.40 \\
\hline $\begin{array}{l}\text { Quadratic } \\
\text { SVM }\end{array}$ & 3 & GA & 99.08 & 99.22 & 98.973 & 98.894 & 98.01 \\
\hline Cubic SVM & 2 & GA & 99.86 & 99.89 & 99.25 & 99.13 & 99.17 \\
\hline $\begin{array}{l}\text { Coarse } \\
\text { Gaussian } \\
\text { SVM }\end{array}$ & 4 & GA & 93.86 & 98.21 & 94.87 & 98.74 & 91.01 \\
\hline $\begin{array}{l}\text { Medium } \\
\text { Gaussian } \\
\text { SVM }\end{array}$ & 21 & No & 96.69 & 98.93 & 96.44 & 98.74 & 94.64 \\
\hline $\begin{array}{l}\text { Fine } \\
\text { Gaussian } \\
\text { SVM }\end{array}$ & 2 & GA & 97.77 & 99.35 & 97.61 & 98.95 & 96.18 \\
\hline
\end{tabular}

In addition, we observed similar findings in the recognition of walking speed for the different walking directions performed by DARwIn-OP. In particular, the performance of the classifiers created for the recognition of gait speed during turning motions is very close. Overall, weighted KNN led to the most proper recognition under the different walking conditions (accuracy > 99.2\%, AUC > 98.94\% MCC $>98.95 \%$ in all cases), and similar behavior was also shown by fine KNN (accuracy $>98.81 \%$, AUC $>97.92 \% \mathrm{MCC}>98.05 \%$ in all cases) and cubic SVM (accuracy $>99.05 \%$, AUC $>98.36 \%$ MCC $>98.75 \%$ in all cases).

The positive results were mainly achieved when the GA selected 2 or 3 features from the 21 determined. This finding highlights the positive contribution of the features selection methods to improve the performance and computational cost of the recognition process [9], [14]. Previous studies also pointed out that the GA, as a wrapper method, is more skilled than PCA to robustly select the relevant features of each locomotion mode without requiring trial-error tests [14]. 


\subsection{Results of Real-Time Phase}

From the findings pointed out in the offline analysis, we specially selected for the real-time recognition the classification models created by weighted $\mathrm{KNN}$, fine KNN, and cubic SVM using the 2 most relevant features picked in offline. These three classification models were also accurate in the real-time recognition of walking directions and speeds (Table 2). The lowest performance was observed in the recognition of gait speed when the DARwIn-OP was walking backwards.

Table 2. Performance obtained in real-time recognition of walking direction and speed.

\begin{tabular}{ccccccc}
\hline $\begin{array}{c}\text { Classification } \\
\text { model }\end{array}$ & $\begin{array}{c}\text { Accuracy } \\
(\boldsymbol{\%})\end{array}$ & $\begin{array}{c}\text { Specificity } \\
(\boldsymbol{\%})\end{array}$ & $\begin{array}{c}\text { Sensibility } \\
\mathbf{( \% )}\end{array}$ & $\begin{array}{c}\text { AUC } \\
(\boldsymbol{\%})\end{array}$ & $\begin{array}{c}\text { MCC } \\
(\boldsymbol{\%})\end{array}$ & $\begin{array}{c}\text { Time } \\
(\mathbf{m s})\end{array}$ \\
\hline Weighted KNN & 99.15 & 99.54 & 99.18 & 99.59 & 98.77 & 89 \\
\hline Fine KNN & 98.92 & 98.96 & 98.93 & 99.04 & 99.01 & 105 \\
\hline Cubic SVM & 98.59 & 98.23 & 98.61 & 99.89 & 97.01 & 129 \\
\hline
\end{tabular}

The results stated in Table 2 outlined that the weighted $\mathrm{KNN}$ is more skilled than fine $\mathrm{KNN}$ and cubic SVM, presenting higher values for the five metrics (e.g. accuracy $=99.15 \%$, AUC $=99.59 \%, \mathrm{MCC}=98.77 \%$ ) studied and a lower elapsed time in the recognition process $(89 \mathrm{~ms})$. By comparing these findings with other ones demonstrated by the real-time recognition tools well established in the literature, we can point out that our strategy exhibits a higher performance (accuracy of 99.15\% versus 90\% [1], 88.8\% [2], 95\% [3]) and a lower time delay ( 89 ms against the delay described in literature that ranges from 300 to $650 \mathrm{~ms}$ [3], [5]) in the recognition of a new locomotion mode. Theses outcomes indicate that the proposed recognition tool is time-effective and suitable for the real-time gait analysis, and can potentially be used to support control strategies in robotics.

\section{Conclusion}

This work contributed to knowledge with the development of a highly accurate, robust and cost-effective tool for the automatic and real-time locomotion mode recognition. From an exhaustive and objective investigation, we verified that $\mathrm{KNN}$ is the most suitable machine learning approach for creating a generalized classification model, which only need to use 2 features to discriminate the different locomotion modes of a DARwIn-OP. The proposed recognition tool showed to be more skilled than the others proposed in the literature. These advances suggest that this tool can potentially be applied in offline or real-time gait analysis, namely in human gait for diagnose or treatment purposes. Future challenges involve the application of the purpose tool in real robotic walkers and 
healthy and pathologic subjects, and the development of strategies to recognize the human's movement intention, which are demanded on neurorehabilitation.

\section{References}

1. M. Goršič, R. Kamnik, L. Ambrožič, N. Vitiello, D. Lefeber, G. Pasquini, and M. Munih, Sensors 14, 2776(2014).

2. A. J. Young, T. A. Kuiken, and L. J. Hargrove, J. Neural Eng. 11,1(2014).

3. H. Huang, F. Zhang, L. J. Hargrove, Z. Dou, D. R. Rogers, and K. B. Englehart, IEEE Trans. Biomed. Eng. 58, 2867(2011).

4. Y. Li and E. Hsiao-Wecksler, Gait mode recognition and control for a portable-powered ankle-foot orthosis (IEEE International Conference on Rehabilitation Robotics, 2013).

5. H. A. Varol, F. Sup, and M. Goldfarb, IEEE Trans. Biomed. Eng. 57, 542(2010).

6. J. Rueterbories, E. G. Spaich, B. Larsen, and O. K. Andersen, Med. Eng. Phys. 32, 545(2010).

7. S. Au, M. Berniker, H. Herr, Neural Networks 21, 654(2008).

8. R. Begg and J. Kamruzzaman, J. Biomech. 38, 401(mar 2005).

9. F. J. Badesa, R. Morales, N. Garcia-Aracil, J. M. Sabater, A. Casals, and L. Zollo, Comput. Methods Programs Biomed. 116, 123(sep 2014).

10. X. Zhang and M. Hashimoto, Mechatronics 22, 33(feb 2012).

11. J. Figueiredo, C. P. Santos, J. L. Pons, and J. C. Moreno, Recognition of Gait Patterns in Human Motor Disorders using Machine Learning: A Review, (2017).

12. A. Samà, C. Angulo, D. Pardo, A. Català, and J. Cabestany, Neurocomputing 74, 2665(sep 2011).

13. J. Wu, J. Wang, and L. Liu, Hum. Mov. Sci. 26, 393(jun 2007).

14. J. Zhang, T. E. Lockhart, and R. Soangra, Ann. Biomed. Eng. 42, 600(mar 2014). 